\title{
Zone Head Selection Algorithm Based on Fuzzy Logic for Wireless Sensor Networks
}

\author{
${ }^{1}$ Anchal Garg, ${ }^{2}$ Gurjinder Kaur \\ ${ }^{1}$ P. G. Scholar, Department of Computer Science and Engineering, Sant Longowal \\ Institute of Engineering and Technology, Longowal, Punjab, 148106 India \\ ${ }^{2}$ Associate Professor, Department of Computer Science and Engineering, Sant \\ Longowal Institute of Engineering and Technology, Longowal, Punjab, 148106 \\ India \\ Ianchal5garg@gmail.com, ${ }^{2}$ gurjinder13@yahoo.com
}

\begin{abstract}
Clustering extends energy resources, improves scalability and preserves communication bandwidth of the network. Clustering is either categorized as static and dynamic or as equal and unequal. Hot-spots issue needs a high overhead and is prone to connectivity problems in the wireless sensor network and this can be only possible because of unequal clustering. In this paper a zone divisional method based on fuzzy logic has been proposed. This method uses a fuzzy logic to form clusters and allot nodes to them for the reduction of energy consumption, and extends the age of the sensor network. The simulation and results section shows that the outperformance of the proposed algorithm, where the (EAUCF) energy-aware unequal clustering fuzzy, (LEACH) lowenergy adaptive clustering hierarchy, (EAMMH) energy-aware multi-hop multi-path hierarchical, and (TTDFP) two-tier distributed fuzzy logic-based protocol for efficient data aggregation in multi-hop wireless sensor networks algorithms. The proposed algorithm has better results in terms of energy consumption minimization, load balancing, and prolongation of the network lifetime.
\end{abstract}

Keywords: Wireless sensor networks, fuzzy logic, hot-spots, energy consumption, network lifetime.

\section{INTRODUCTION}

The wireless sensor network has recently become a point of interest for many researchers due to their great usefulness in many fields such as military, medical and environmental sciences, disaster prediction, manufacturing industry, traffic management etc. WSN is a set of small sensor nodes with low-cost and low power, connected by wireless links and distributed over area. Those sensor nodes can collect data from the surroundings, process data, and communicate with each other to root the collected data to the base station (BS). Due to dense deployment and unattended environments the sensors are usually equipped with small batteries and it cannot be recharged [1]. WSN is divided into a group of clusters, each of which has manager called the cluster head $(\mathrm{CH})$. The sensed data collected by cluster member (CM) nodes sends directly to corresponding $\mathrm{CH}$ and then cluster head send data to BS. The responsibilities of the CHs are aggregating data from CMs and sending it to BS [2]. In WSN clustering is proposed to achieve high energy efficiency and 
improve scalability. The hotspot problem is occurring while using multi-hop communication the sensor nodes does not send the sensed data directly to the base station but they send data with a multi-hop approach. The nodes which are near to the BS have to communicate with their own sensed data and should also forward data from other nodes. This indicates that the reduction of their energy quicker than other sensor nodes. The unequal clustering methods are usually presented in which the sensor network is separated into clusters with unequal sizes. Using unequal clustering algorithms for solving this hotspot issue [3], [4]. Fuzzy Logic is based on heuristic knowledge and human reasoning which is one of the best problems solving allowing a time-consuming latency and prolongs lifetime of the network. In this formation of cluster each non-CH nodes calculates a chance value for being connected to each $\mathrm{CH}$ by applying fuzzy logic, using closeness to $\mathrm{CH}$ and residual energy as fuzzy inputs [5]. Fuzzy Logic is one such computational intelligence (CI) used for choosing $\mathrm{CH}$ nodes in the WSN. Fuzzy Logic mostly consists of four huge components. It contains a Fuzzifier, a Rule Base, an Inference Engine and a Defuzzifier. The input values are usually crisp values which again are converted into fuzzy linguistic variables. The fuzzified values are transferring to Fuzzy Decision Block (FDB). FDB is composed of Inference Engine and Rule Base. It presents fuzzy output mostly based on the fuzzy rules. Then the fuzzy output is transformed into crisp output by means of defuzzification [6].

The remainder of this paper is organized as follows: Section 2 reviews the related work, Section 3 describes the proposed protocol method in the detail and Section 4 discusses the simulation results. Section 5 concludes the paper.

\section{RELATED WORK}

Batra and Kushwah [7] proposed Fuzzy Logic based Cluster Head Selection (FLCHS) method helps in the load balancing and energy consumption minimization. In this work FLCHS is compared with the well-known Stable Election Protocol for heterogeneous networks. FLCHS protocol performs $12 \%$ better than SEP protocol in terms of network lifetime, energy consumption and throughput. The FLCHS algorithm produces optimal number of clusters and selects cluster head with high energy. Kongara et al. [8] proposed Dynamic Cluster Formation Mechanism (DCFM) to increase the lifetime of Wireless Sensor Networks by using fuzzy logic-based clustering approach. In this work the proposed DCFM approach is compared with the LEACH algorithm. Simulation result show that the proposed DCFM approach performs better result than LEACH algorithm. Akila and Venkatesan [9] proposed a geo-cluster head set approach to address the hot-spot problem in WSNs. A geo-cluster head set method handles the hot-spot problem and the load is balanced between the sensor nodes of the network in terms of enhance the network's lifespan. The result section show the performance of the proposed Zonal Clustering Algorithm was compared with other three approaches LEACH, EACLE and EBCAG. Proposed method ZCA has best results than other existing approaches. Abidi and Ezzedine [10] proposed a fuzzy $\mathrm{CH}$ election algorithm based on LEACH protocol. This proposed algorithm uses Fuzzy Logic method to elect cluster head. The simulation results show that proposed approach is effective to increase the lifespan of the network and reduce energy consumption better than LEACH, CHEF and EAUCF. El Alami and Najid [11] proposed an enhanced clustering hierarchy (ECH) to extend the energy efficiency of sensor network and enhance network lifetime. This algorithm is implemented in the both homogeneous network and heterogeneous network. Simulation results show that proposed algorithm ECH is more efficient than other routing protocols. Yadav et al. [12] proposed Energy Efficient Probabilistic Clustering Technique (EEPCT) to improve the energy efficiency of probabilistic clustering algorithms by optimizing the number of clusters and well distribution of $\mathrm{CHs}$ across the network in each round. This work has more focus on probabilistic clustering algorithms. The simulation result section displays the outperformance of proposed method is significant improvement in energy efficiency of probabilistic clustering protocols and prolongation of network lifespan compared to LEACH, HEED and SEP algorithms.

These above protocols are proposed for multi-hop transmission and clustering which are based on 
fuzzy logic approach Moreover, some protocols are proposed for cluster head selection algorithms. Most of these protocols are based on cluster head selection method which uses fuzzy logic approach. This consumes a very large amount of system energy due to its high complexity. For reducing this high usage and complexity proposed an algorithm namely a zone divisional method based on fuzzy logic to minimize the consumption of energy, load is balanced, and increase lifetime of the sensor network.

\section{Methodology}

This methodology is useful to attain the aims of the study. This method is all about a zone divisional method based on fuzzy logic which uses unequal clustering based on fuzzy logic approach. In this paper the proposed algorithm improves cluster header selection method. In this section the detailed methodology is given below.

1. Choose an area dimension. This will be the whole area in which all the communication will happen in a well monitored manner.

2. Deploy sensor network nodes in that area, in a random manner. Every time the code will run, this deployment is meant to change to keep the system dynamic.

3. All the transfer of information from source to the receiver which will coordinate by the base station. Each route will be configured on the basis of base station.

4. The whole area is divided into zones like nine zones in our case. Zones are very essential and each zone will have its own head coordinator.

5. There will be some sensor nodes available in each zone.

6. Our task is to choose the zone header which will coordinate all the information or data transfer from/to that specific zone.

7. Create clusters and assign nodes to them by using fuzzy logic.

8. This process use high system energy and is very complex for the system architecture if the number of sensor nodes are very high. We have to reduce this high usage and complexity for efficient data flow.

9. Therefore, our proposed method will select the zone header but we will be more concerned on saving system energy and decreasing the complexity.

10. This will be easy to deploy for sensor networks with large number of sensor nodes as that would be case where maximum system energy will be used.

11. By the use of MATLAB the whole system will be simulated.

12. The different parameters will be calculated to test the system efficiency and will be compared with the previously computed parameters.

\section{Flow Chart}




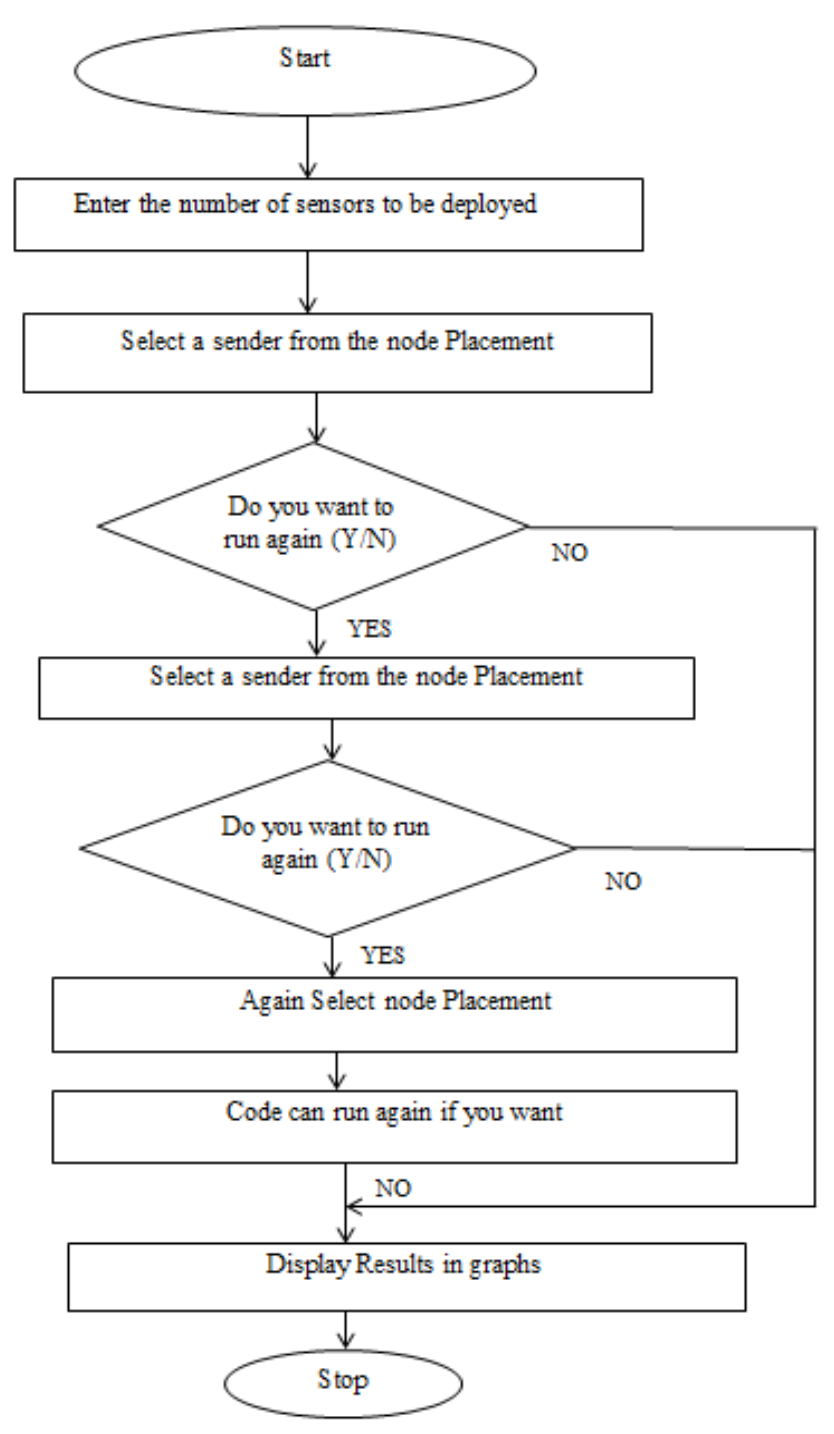

Fig. 1 Flow Chart of Methodology

\section{SIMULATION RESULTS}

The proposed method has been evaluated by using MATLAB because its Fuzzy Toolbox notices all fuzzy membership functions; hence it is appropriate for use. This proposed method was simulated by using MATLAB. The following are simulation results of the proposed algorithm step by step implementation and results have been shown.

These graphs show the final results of the energy consumption after every iteration. 


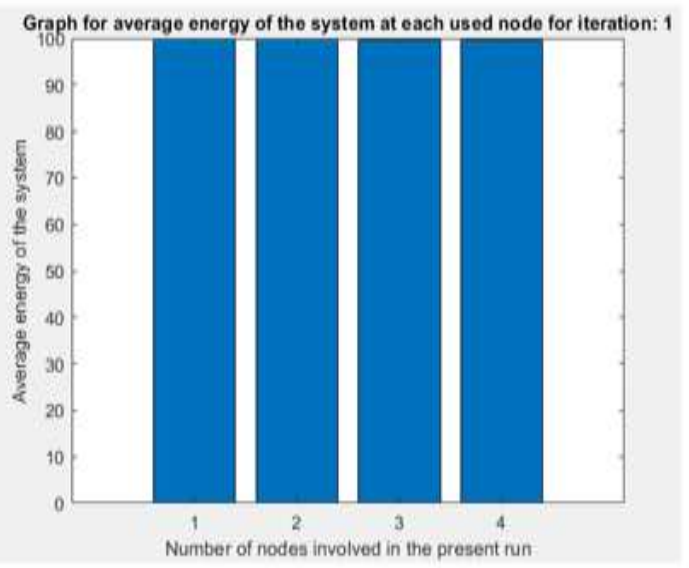

Fig. 2 Graph for average energy of the system at each used node for iteration: 1 (average energy $V s$ no. of nodes involved in the present run)

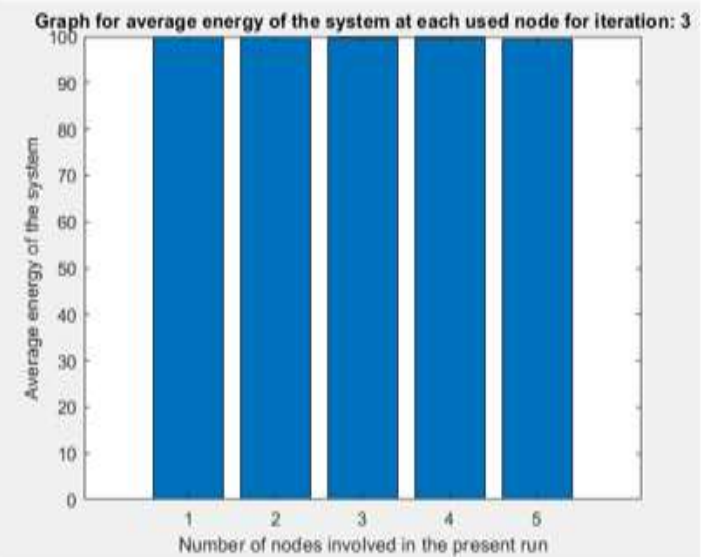

Fig. $4 \mathrm{Graph}$ for average energy of the system at each used node for iteration: 3 (average energy Vs no, of nodes involved in the present run)

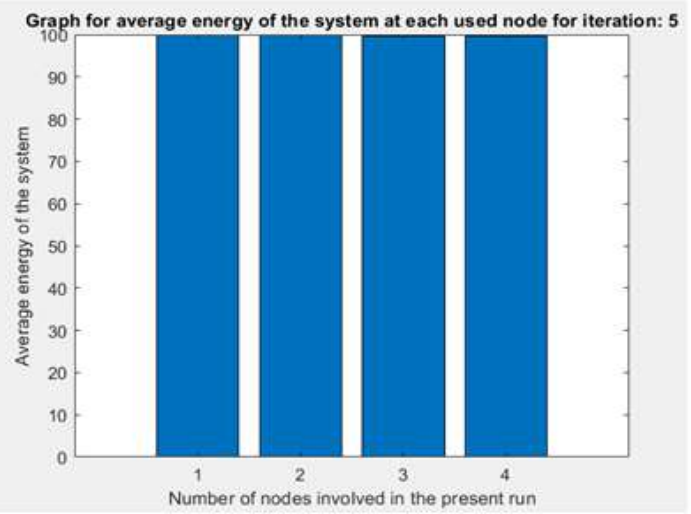

Fig. $6 \mathrm{Graph}$ for average energy of the system at each used node for iteration: 5 (average energy Vs no. of nodes involved in the present run)

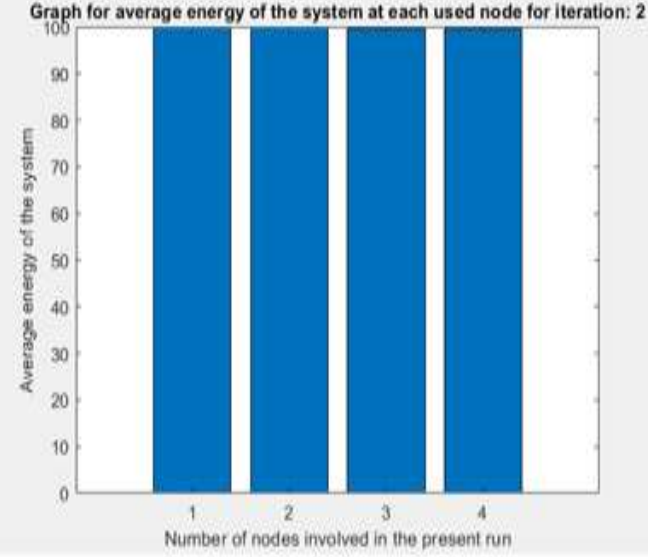

Fig, $3 \mathrm{Graph}$ for average energy of the system at each used node for iteration: 2 (average energy $V$ s no. of nodes involved in the present run)

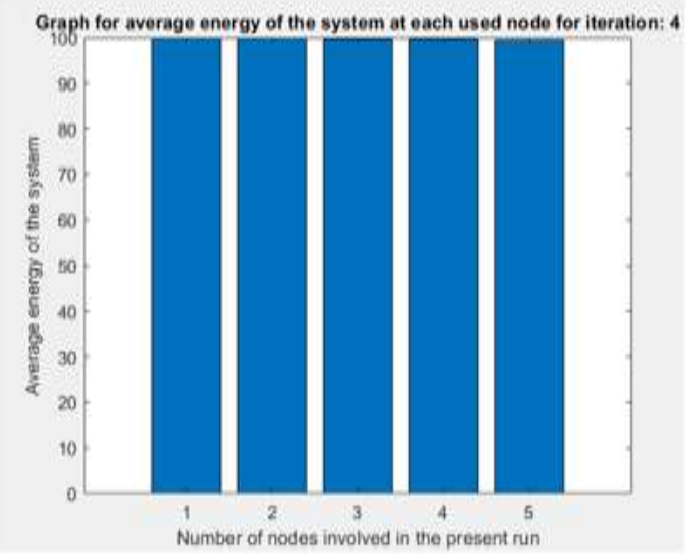

Fig. 5 Graph for average energy of the system at each used node for iteration: 4 (average energy $V s$ no. of nodes involved in the present run)

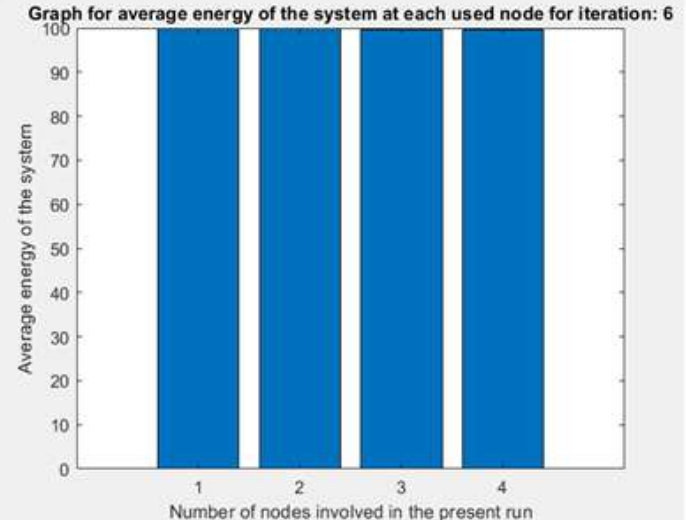

Fig. 7 Graph for average energy of the system at each used node for iteration: 6 (average energy Vs no. of nodes involved in the present run) 


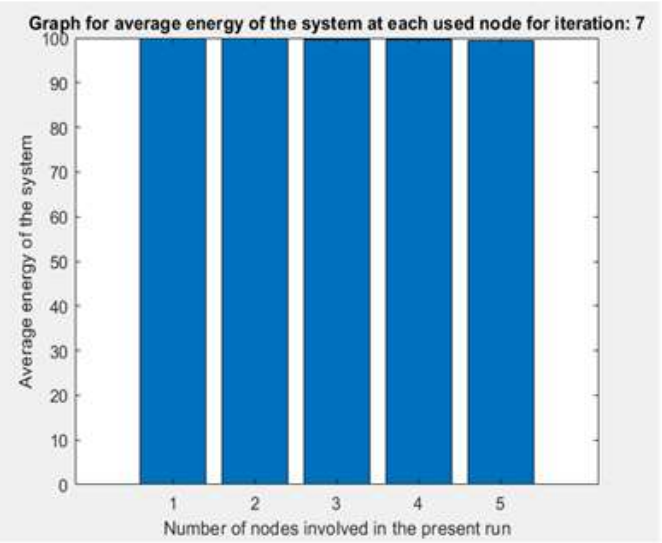

Fig. 8 Graph for average energy of the system at each used node for iteration: 7 (average energy Vs no. of nodes involved in the present run)

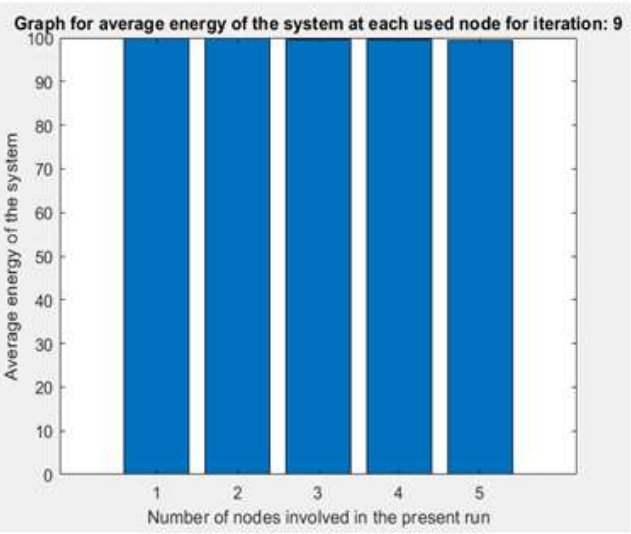

Fig. $10 \mathrm{Graph}$ for average energy of the system at each used node for iteration: 9 (average energy Vs no. of nodes involved in the present run)

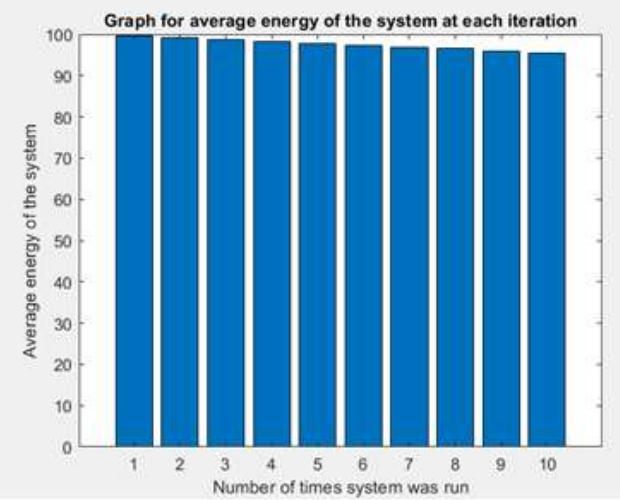

Fig. 12 Graph for average energy of the system at each iteration (average energy Vs no. of times system was run)

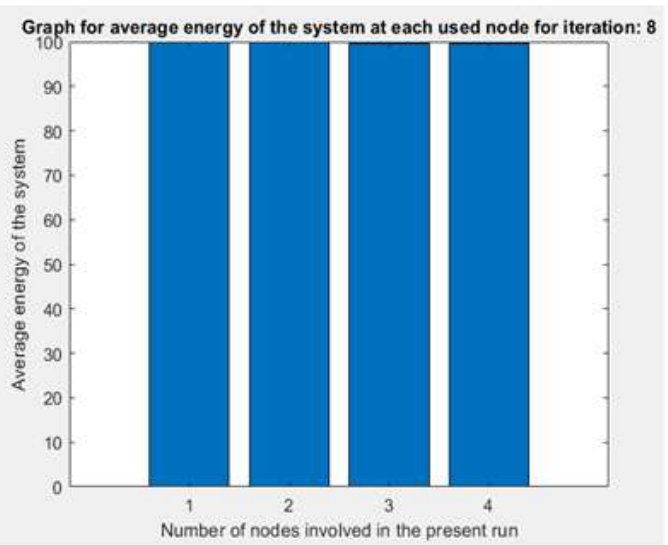

Fig. 9 Graph for average energy of the system at each used node for iteration: $\mathbf{8}$ (average energy Vs no. of nodes involved in the present run)

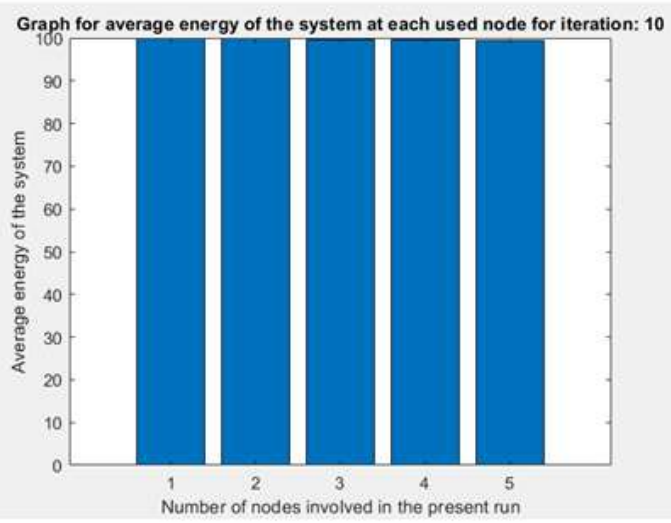

Fig. $11 \mathrm{Graph}$ for average energy of the system at each used node for iteration: 10 (average energy Vs no. of nodes involved in the present run)

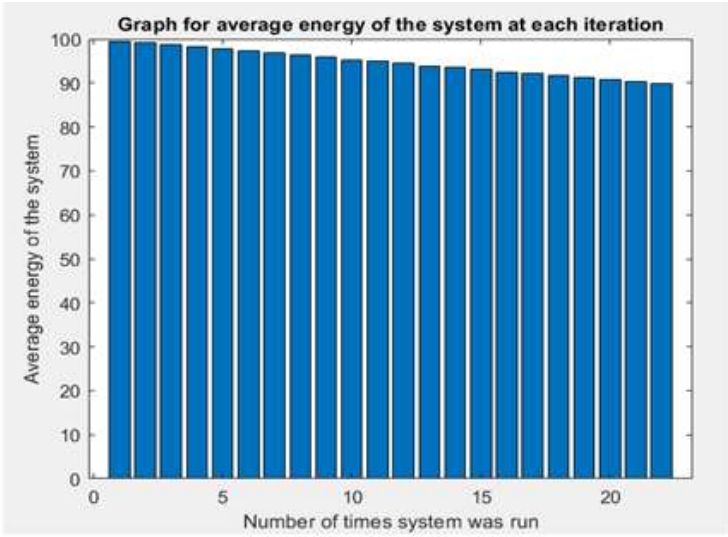

Fig. 13 Graph for average energy of the system at each iteration (average energy Vs no. of times system was run) 


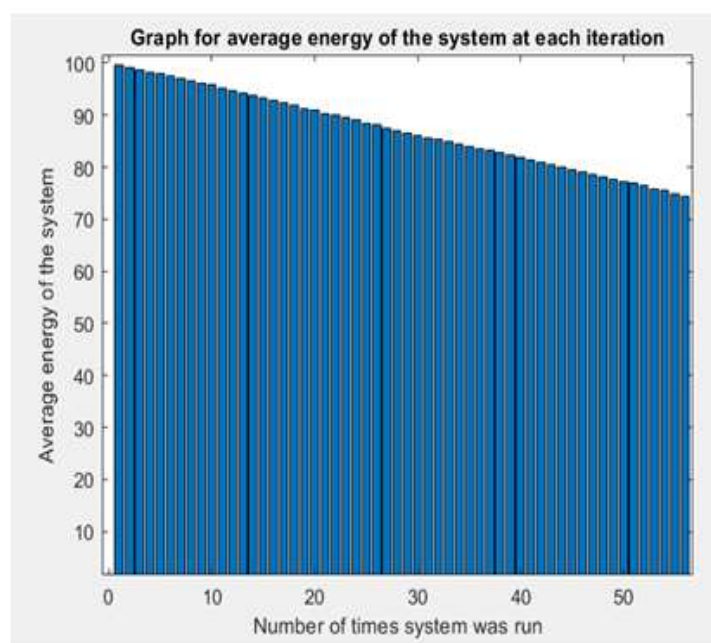

Fig. 14 Graph for average energy of the system at each iteration (average energy Vs no. of times system was run)

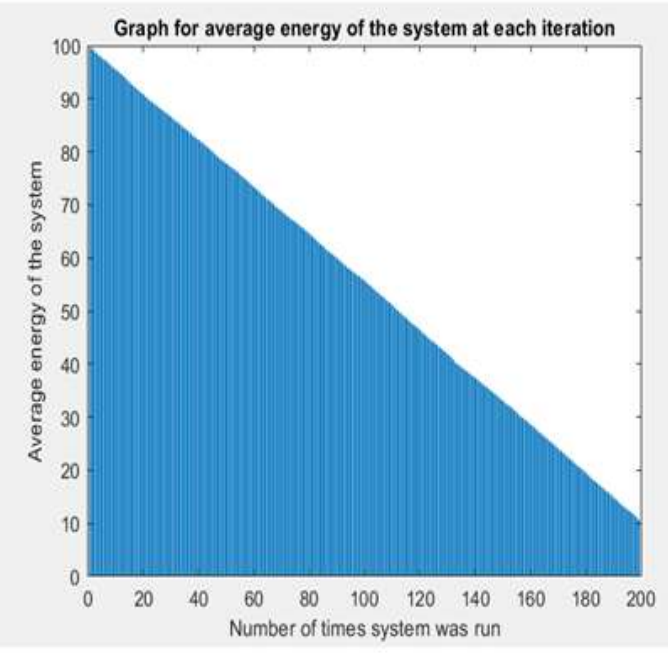

Fig. $16 \mathrm{Graph}$ for average energy of the system at each iteration (average energy Vs no. of times system was run)

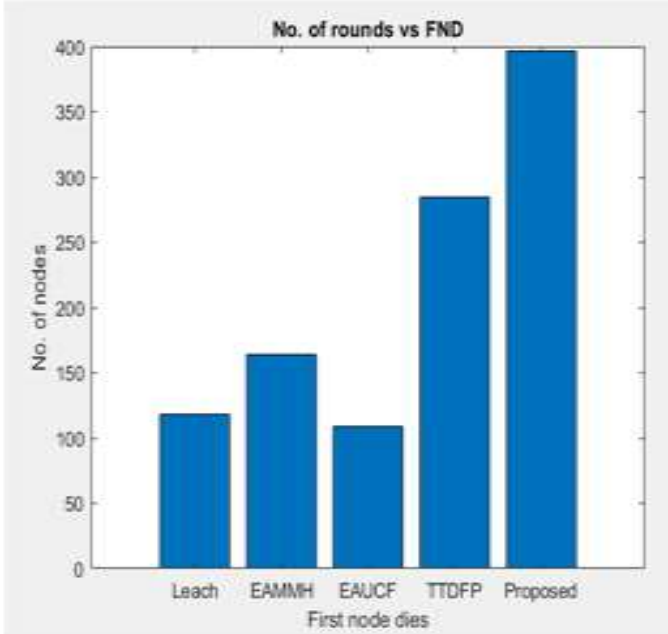

Fig. 18 No. of rounds vs First Node Dies

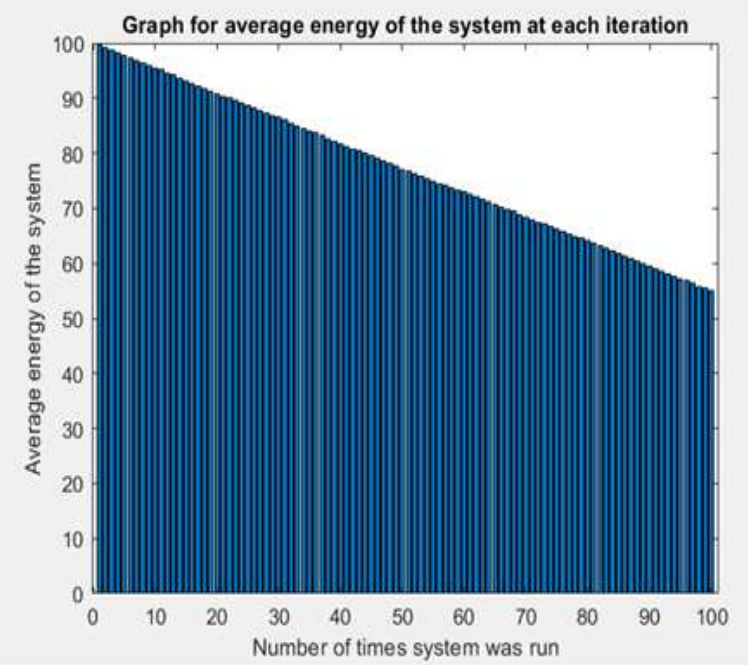

Fig. 15 Graph for average energy of the system at each iteration (average energy Vs no. of times system was run)

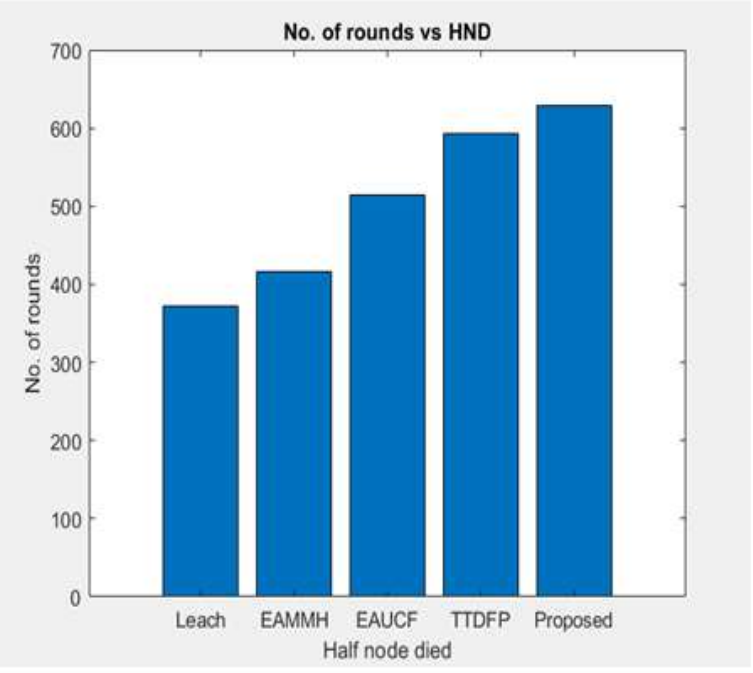

Fig. 17 No. of rounds vs Half Node Died

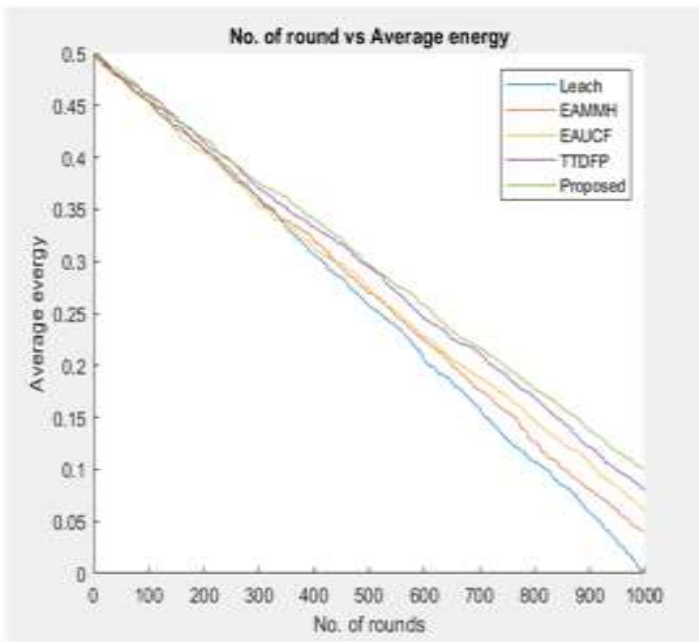

Fig. 19 No. of round vs Average energy 
Table 1. Average energy of the system at each iteration

\begin{tabular}{|c|c|}
\hline $\begin{array}{c}\text { No. of times } \\
\text { system was run }\end{array}$ & $\begin{array}{c}\text { Average } \\
\text { energy of the } \\
\text { system }\end{array}$ \\
\hline 1 & 99.6 \\
\hline 2 & 99.2 \\
\hline 3 & 98.7 \\
\hline 4 & 98.2 \\
\hline 5 & 97.8 \\
\hline 6 & 97.4 \\
\hline 7 & 96.9 \\
\hline 8 & 96.5 \\
\hline 9 & 96 \\
\hline 10 & 95.5 \\
\hline 22 & 94.6 \\
\hline 56 & 87 \\
\hline 100 & 77.1 \\
\hline 199 & 55.3 \\
\hline
\end{tabular}

\section{CONCLUSION}

In this paper we proposed a zone divisional method based on fuzzy logic approach because it performs better than other comparing algorithms in terms of load is balanced, saving more energy; reducing the complexity of sensor nodes and prolongation of network lifespan. In the wireless sensor networks unequal clustering has a high overhead and is prone to connectivity problems. The simulation and results section displays that the proposed algorithm is compared with the other four algorithms namely LEACH, EAMMH, EAUCF and TTDFP the proposed algorithm has better results in terms of energy consumption minimization, load balancing, and prolongs the network survival time.

\section{REFERENCES}

[1] Y. El Assari, S. Al Fallah, J. El Aasri, M. Arioua, and A. El Oualkadi, "Energy-efficient multi-hop routing with unequal clustering approach for wireless sensor networks," Int. J. Comput. Networks Commun., vol. 12, no. 3, pp. 55-73, 2020, doi: 10.5121/ijcnc.2020.12304.

[2] F. Liu and Y. Chang, "An Energy Aware Adaptive Kernel Density Estimation Approach to Unequal Clustering in Wireless Sensor Networks," IEEE Access, vol. 7, pp. 40569-40580, 2019, doi: 10.1109/ACCESS.2019.2902243.

[3] K. Sundaran, V. Ganapathy, and P. Sudhakara, "Fuzzy logic based Unequal Clustering in wireless sensor network for minimizing Energy consumption," Proc. 2017 2nd Int. Conf. Comput. Commun. Technol. ICCCT 2017, pp. 304-309, 2017, doi: 10.1109/ICCCT2.2017.7972283.

[4] Y. Tao, J. Zhang, and L. Yang, “An unequal clustering algorithm for wireless sensor networks based on interval type-2 tsk fuzzy logic theory," IEEE Access, vol. 8, pp. 197173-197183, 2020, doi: 10.1109/ACCESS.2020.3034607.

[5] M. Fattoum, Z. Jellali, and L. N. Atallah, "Fuzzy Logic-based Two-Level Clustering for Data Aggregation in WSN," Proc. 17th Int. Multi-Conference Syst. Signals Devices, SSD 2020, pp. 360 365, 2020, doi: 10.1109/SSD49366.2020.9364181.

[6] V. Rajaram, "Fuzzy Logic b ased Unequal Clustering i n Wireless Sensor Networks f or Effective Energy Utilization,” pp. 2056-2061, 2017.

[7] P. K. Batra and R. Kushwah, "Fuzzy Logic based Cluster Head Selection method for Heterogeneous Wireless Sensor Networks," Proc. IEEE Int. Conf. Image Inf. Process., vol. 2019-Novem, pp. 86-90, 2019, doi: 10.1109/ICIIP47207.2019.8985903.

[8] M. C. Kongara, V. Kuppili, and D. R. Edla, "Dynamic cluster formation mechanism in wireless sensor networks using fuzzy logic," Proc. - 2019 Int. Conf. Appl. Mach. Learn. ICAML 2019, pp. 291-295, 2019, doi: 10.1109/ICAML48257.2019.00061. 
[9] I. S. Akila and R. Venkatesan, "An energy balanced geo-cluster head set based multi-hop routing for wireless sensor networks," Cluster Comput., vol. 22, no. s4, pp. 9865-9874, 2019, doi: 10.1007/s10586-018-1724-Z.

[10] W. Abidi and T. Ezzedine, "Fuzzy Cluster Head Election algorithm based on LEACH protocol for wireless sensor networks," 2017 13th Int. Wirel. Commun. Mob. Comput. Conf. IWCMC 2017, pp. 993-997, 2017, doi: 10.1109/IWCMC.2017.7986421.

[11] H. El Alami and A. Najid, "ECH: An Enhanced Clustering Hierarchy Approach to Maximize Lifetime of Wireless Sensor Networks," IEEE Access, vol. 7, pp. 107142-107153, 2019, doi: 10.1109/ACCESS.2019.2933052.

[12] R. K. Yadav, D. Gupta, and D. K. Lobiyal, "Energy Efficient Probabilistic Clustering Technique for Data Aggregation in Wireless Sensor Network," Wirel. Pers. Commun., vol. 96, no. 3, pp. 40994113, 2017, doi: 10.1007/s11277-017-4370-5. 